that normally processes nociceptive input. Back filling of the retina from the thalamic nuclei, combined with immunohistochemistry, revealed the identity of these ipRGCs that project to the ventro-posterior thalamus. Electrophysiological recording of dural-driven nociceptive cells in animal experiments clearly showed that light was able to modulate the firing patterns of these cells, providing the functional linkage between the visual and sensory inputs. Although a more detailed analysis of the response characteristics and stimulus parameters in this interaction is required, the primary finding of an interaction was clear. To further establish their findings, the authors used juxtacellular dye labeling of physiologically characterized nociceptive/visually modulated thalamic cells, combined with anterograde retinal afferent labeling, to show retinal afferent innervation of these thalamic cells. Although only four thalamic cells were labeled in this way, the intimate relationship between the retinal terminals and thalamic neurons was clear. The juxtacellular-labeling technique also allowed thalamic projections to the cortex to be traced. These projections were not particularly well localized and the rather widespread terminations in multiple cortical regions limit any real understanding of how higher-order processing of this light-modulated nociceptive information might occur. As there was no quantification of these findings, it is hard to relate the demonstrably retinorecipient nociceptive thalamic neurons to specific cortical projection patterns.

The final part of this study attempts to link thalamic drive to cortical processing, and although the results are tantalizing, they are still incomplete. It is also not at all clear how thalamic input from a relatively small number of cells found in rats truly applies to humans, although the authors suggest that this input is the basis of the intense pain response associated with photophobia in migraine ${ }^{13}$. However, in the absence of a link between thalamic and cortical pain processing regions, it is still unclear what role these thalamic neurons might have in terms of cortical projections and pain perception. The rather diffuse thalamic projections to multiple cortical regions do not help in understanding how such information is processed at higher levels or in resolving the potential substrates for pain perception. It may be that the assumption that rats, as do humans, suffer from migraine is an overambitious one ${ }^{14}$.

These results therefore still leave many questions unanswered and provide much fodder for future work. For example, it would be interesting to tune the illumination to the physiological response properties of the ipRGCs ${ }^{10,15}$ rather than being delivered at very low and very high intensities (500 lx and 50,000 lx). Such stimulation could provide even better evidence for the nature of the modulatory role of retinal afferents onto thalamic neurons by showing that thalamic response properties correlate well with properties of the ipRGCs. In the same vein, the future discovery of wavelength specific effects on partially sighted migraine sufferers might provide a relatively easy therapeutic approach to treat photo-exaggerated migraine. Would these patients show any form of selective wavelength-specific effects of light in terms of the light associated exacerbation of their migraine? These results suggest that an imaging study looking at the effects of differential illumination of migraine sufferers would be, quite literally, illuminating.

1. Noseda, R. et al. Nat. Neurosci. 13, 239-245 (2010). 2. Mayberg, M.R., Zervas, N.T. \& Moskowitz, M.A. J. Comp. Neurol. 223, 46-56 (1984).

3. Keller, J.T., Saunders, M.C., Beduk, A. \& Jollis, J.G. Brain Res. Bull. 14, 97-102 (1985).

4. Craig, A.D. Annu. Rev. Neurosci. 26, 1-30 (2003).

5. Tracey, I. \& Mantyh, P.W. Neuron 55, 377-391 (2007).

6. Perl, E.R. Nat. Rev. Neurosci. 8, 71-80 (2007).

7. May, A. Nat. Rev. Neurol. 5, 199-209 (2009).

8. Goadsby, P.J., Charbit, A.R., Andreou, A.P., Akerman, S \& Holland, P.R. Neuroscience 161, 327-341 (2009)

9. Masland, R.H. Nat. Neurosci. 4, 877-886 (2001).

10. Hattar, S., Liao, H.W., Takao, M., Berson, D.M. \& Yau, K.W. Science 295, 1065-1070 (2002).

11. Hattar, S. et al. J. Comp. Neurol. 497, 326-349 (2006).

12. Lucas, R.J., Douglas, R.H. \& Foster, R.G. Nat. Neurosci. 4, 621-626 (2001).

13. Mogil, J.S. Nat. Rev. Neurosci. 10, 283-294 (2009).

14. Craig, A.D. Nat. Rev. Neurosci. 10, 466 (2009).

15. Tu, D.C. et al. Neuron 48, 987-999 (2005).

\title{
Amongst equals
}

Personality differences among individuals can greatly influence how they divide resources. For example, some people are more averse to inequalities in resource provision than others. A study by Haruno and Frith on pp. 160-161 of this issue suggests that emotion, rather than conscious deliberation, drives these social preferences about reward distribution.

Haruno and Frith asked subjects to choose between three options, with each option specifying different combinations of rewards for themselves and an anonymous partner. Some subjects' choices were only influenced by how much reward there was for themselves, but others preferred reward pairs in which the difference between the reward for themselves and their partner was minimal. Previous work has shown that these differences between the former group of 'individualists' versus the latter 'prosocial' subjects are stable over many years and predict real-life decisions.

But how do these differences arise? One theory suggests that the automatic, emotion-driven impulse is to be selfish and maximize gains for oneself, but slower, conscious deliberation between alternative courses

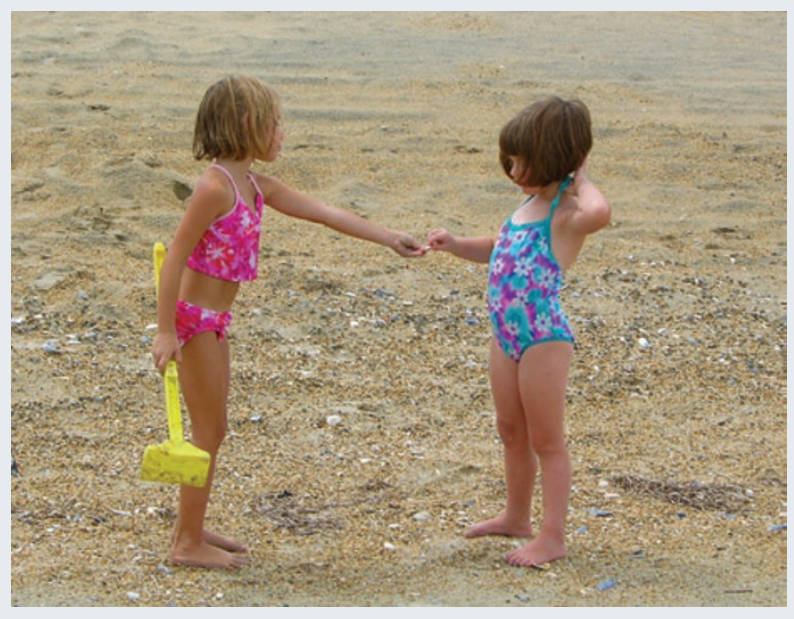
of action can overcome this automatic impulse. This theory would predict that individualist and prosocial subjects would differ in the extent of the conscious decision-making process, which is usually thought to be localized to frontal areas (such as the prefrontal cortex).

To test this idea, the authors used functional magnetic resonance imaging to track changes in activation as prosocial and individualist subjects rated the desirability of reward pairs, with each pair specifying a reward for the subject and their partner. Prosocial subjects had greater amygdala activation than individualists. Furthermore, amygdala activation in the prosocials during each reward pair presentation correlated with the reward difference between the subject and the partner. The authors therefore conclude that social preferences about how rewards should be divided are not driven by top-down, conscious deliberation, but instead reflect automatic emotional processing.

Charvy Narain 